\title{
VALORACIONES DE FUTUROS PROFESORES DE ESPAÑOL HACIA LAS VARIEDADES CULTAS DE SU LENGUA. DATOS DE GRANADA
}

\section{EVALUATIONS OF FUTURE SPANISH TEACHERS TOWARDS THE VARIETIES OF THEIR LANGUAGE. GRANADA DATA}

\author{
Antonio Manjón-Cabeza Cruz \\ Universidad de Granada \\ amanjoncabeza@ugr.es \\ https://orcid.org/0000-0002-2112-3793
}

Recibido: $15 / 10 / 2019$

Aceptado: 30/01/2020

\section{Resumen}

Tras la aplicación de la encuesta de PRECAVES XXI, comprobamos que los futuros profesores de español granadinos muestran un grado de identificación de las variedades que oscila entre la buena identificación (variedades rioplatense, castellana y andaluza), media identificación (canaria, caribeña, mexicana y andina) y mala identificación (chilena).

Constatamos la tendencia a establecer jerarquías entre variedades, en este caso a favor de la castellana. Asimismo, las valoraciones directas nos señalan que la variedad castellana tiene un tratamiento especial frente a las demás, porque tiene la mejor consideración cognitiva (es considerada urbana y clara), pero tiene la peor consideración afectiva porque se considera lejana, fea y aburrida. Las valoraciones indirectas de la variedad andaluza reflejan un fuerte grado de estigmatización, especialmente en la valoración socioeconómica. También hay una variedad americana mal valorada: la andina. Suponemos

\begin{abstract}
After the aplication of the PRECAVES XXI survey, we checked that the future granadan Spanish teachers show a grade of identification of the varieties that oscillate between the good identification (varieties from Rio de la Plata, Castilian and Andalucian), médium identification (Canarian, Caribean, Mexican and Andean) and bad identification (Chilean).

We verified the tendency to establish hierarchies between varieties, in this case in favor of the Castillian. Additionally, the direct valuations point us that the Castillian variety has a special treatment than the others, because it has a better cognitive consideration (is considered urban and clear), but it has the worst afective consideration because it is considered distant, ugly and boring. The indirect valuations of the Andalucian varieties show a strong grade of stigmatisation, specially in the socieconomic valuation. There is also an American variety badly valued: the Andean. We suppose that a
\end{abstract}

Para citar este artículo / To cite this article: Manjón-Cabeza Cruz, Antonio (2020). Valoraciones de futuros profesores de español hacia las variedades cultas de su lengua. Datos de Granada. ELUA, 34: 131-152. https://doi.org/10.14198/ELUA2020.34.6

Enlace / Link: https://doi.org/10.14198/ELUA2020.34.6 
que se refleja una reacción discriminatoria hacia comunidades inmigrantes de ese origen.

PALABRAS CLAVE: Creencias y actitudes lingüísticas; variedades del español; PRECAVES XXI; Granada (España); futuros profesores de español. discriminatory reaction towards the immigrant communities from that origin is reflexed.

KEYWORDS: Beliefs and linguistics atittudes; Spanish varieties; PRECAVES XXI; Granada (Spain); future Spanish teachers.

\section{INTRODUCCIÓN}

El estudio ${ }^{1}$ de las creencias y actitudes de los hablantes hacia fenómenos lingüísticos de diversa índole ha sido un campo básico de la sociolingüística. Esto queda demostrado con la consulta de los manuales al uso que no dejan de dedicar capítulos, generalmente extensos, a esta parcela de investigación (López Morales 1989; Moreno Fernández 1998; Silva-Corvalán 2001; Almeida 2003, etc.). Otro camino para comprobar la importancia de esta parcela consiste en cerciorarse de que todas las escuelas sociolingüísticas no han dejado de tratar el tema porque, como señala García Marcos (2015: 85), el estudio de las valoraciones subjetivas es "un capítulo apasionante de la sociolingüística que ha tenido una extraordinaria relevancia, no solo por su interés intrínseco en el ámbito descriptivo, sino también por su proyección a otras áreas de la sociolingüística, así como por sus decisivas aplicaciones".

Aunque han sido muchos los acercamientos al estudio de las valoraciones subjetivas, de manera general, como señala Santana Marrero (2018a: 74), "pueden establecerse dos formas de concebir estos conceptos que, a su vez, han generado estrategias de trabajo diferenciadas: concepción conductista y concepción mentalista".

Para esta investigación seguimos las pautas metodológicas del Proyecto para el Estudio de las Creencias y Actitudes hacia las Variedades del Español del siglo XXI (PRECAVES XXI), en el que colaboran 20 equipos de investigadores de ambos lados del Atlántico, como se puede comprobar en http://www.variedadesdelespanol.es/.

Este proyecto adopta un planteamiento cognitivo o mentalista (Cestero Mancera y Paredes García 2015), que conlleva que estudiemos las actitudes a partir de las reacciones que suscitan en los sujetos una serie de estímulos lingüísticos. Según López Morales (1989: 291), las creencias pueden dividirse en dos bloques: afectivas, que conectan con apreciaciones emocionales hacia la realidad lingüística objeto de estudio: sentimientos que se relacionan con gustos, identidad, solidaridad, lealtad, etc. y cognitivas, relacionadas con los propios conocimientos de los que parte el hablante, es decir, de la conciencia sociolingüística basada en prestigios abiertos, sociales y lingüísticos.

Aunque los grupos objeto de investigación en este campo pueden ser muy variados, como se puede comprobar en la amplia nómina expuesta en Cestero Mancera y Paredes García (2018a: 16-17), nuestro interés está en un grupo transmisor de modelos lingüísticos prestigiosos, puesto que sus valoraciones tendrán repercusiones en el conjunto social, ya que, si seguimos a Moreno Fernández (1998: 189), "Se podría definir el prestigio como un proceso de concesión de estima y respeto hacia individuos o grupos que reúnen ciertas

1 Este estudio se ha realizado en el marco del Proyecto ECOPASOS-Granada financiado por MINECO/FEDER (Ref. FFI2015-68171-C5-2-P), integrado en el proyecto PRESEEA (Proyecto para el estudio sociolingüístico del español de España y América). 
características y que lleva a la imitación de las conductas y creencias de esos individuos o grupos".

Es problemático establecer una nómina fiable de esos grupos prestigiados, puesto que pueden ir desde los cantantes populares hasta los profesionales de la medicina. Un acercamiento restrictivo, como el de García Marcos y Fuentes González (1996), seleccionó cuatro grupos profesionales: docentes, periodistas, juristas y religiosos.

Nos centraremos en el colectivo docente, pero en un subgrupo que consideramos especialmente importante: los futuros profesores de español, tanto en su consideración como lengua materna, como en su vertiente como lengua extranjera. Por esto, la muestra ha estado constituida en su totalidad por jóvenes universitarios granadinos de Grado en Filología Hispánica. Aunque este grado no tiene un perfil profesionalizante dirigido a la docencia, en la práctica estos alumnos cubren con preferencia la docencia de las distintas materias de lengua y literatura españolas en educación secundaria y la enseñanza del español como lengua extranjera. El horizonte profesional de la mayoría de estos jóvenes está, pues, relativamente delimitado.

Además, se trata de futuros profesores insertos en una de las variedades del español que podemos calificar de más problemáticas desde el punto de vista de las actitudes y creencias: el andaluz.

Es sabido, que tras la fijación de la capitalidad en Madrid, y por avatares sociohistóricos diversos, el proceso de normalización lingüística llevó a que, en el español de Europa, el estándar fuese el de Castilla. Se inició así un proceso de estigmatización de las otras variedades europeas que llega a nuestros días.

La estigmatización de los rasgos andaluces tiene dos vertientes:

a) Hay procesos de estigmatización interna, puesto que los andaluces estigmatizan ciertas variantes por la actuación de diversas valoraciones (actitudes y creencias). De los muchos rasgos que han sufrido este tipo de proceso recordemos ahora el llamado ceceo, el heheo o la variante palatal fricativa $/ \int /$. Ni qué decir tiene que todos los rasgos con estigmatización interna cuentan además con estigmatización externa.

b) Existen también procesos de estigmatización externa, es decir, hay fenómenos que en andaluz no son valorados negativamente, pero sobre los que pesan valoraciones negativas por parte de usuarios de otras variedades. Es el caso de la pérdida de la /-s/ o de la concordancia ustedes $+2^{a}$ persona del plural, propia del oeste andaluz.

Prueba de estas consideraciones negativas son las calificaciones dadas al andaluz por los madrileños, como reflejan los datos de Yraola (2014) o los toledanos, como se puede consultar en Manjón-Cabeza (2000) o Crespo y Manjón-Cabeza (1996).

La estigmatización, unida a la cercanía geográfica con la variedad estándar y a los modernos procesos de globalización o influencia de los medios de comunicación masivos está llevando a un proceso de convergencia (Villena Ponsoda, 2006) con el estándar, o proceso de asimilación como quieren otros (Moreno Cabrera, 2000), que tiende a acentuar las consideraciones negativas hacia esta variedad. Hay que recordar que los procesos de convergencia están más avanzados en la parte oriental de Andalucía que en la occidental.

Esta situación ha llevado al andaluz a ser objeto de numerosísimos estudios, incluyendo recopilaciones bibliográficas como las de Mondéjar (2006) quien anotó 1428 publicaciones. 
En esos estudios no faltan los que tratan las actitudes y creencias, casi siempre con pretensiones de repercusión posterior en estudios de política y planificación lingüística, entre los que podemos citar a Alcaraz et al. (1979), Vaz de Soto (1981), De Molina Redondo (1985) García Marcos y Manjón-Cabeza (1989), Fuentes González (1996), Martínez y Moya (2000), Díaz Salgado (2002), Carbonero (2003), García Marcos (2008), y un largo etcétera. Sin embargo, muy pocos de estos estudios se centran en el ámbito de la educación, como hacemos en este trabajo.

Queda, pues, patente nuestro objetivo principal: el estudio de las evaluaciones hacia las variedades del español de futuros profesores de español de origen andaluz, concretamente granadino. Para conseguir ese objetivo debemos responder a las siguientes preguntas: ¿Los futuros profesores de español establecen jerarquías entre variedades? ¿Se identifican satisfactoriamente las distintas variedades estudiadas? ¿Hay diferencias entre las evaluaciones cognitivas y afectivas? ¿Las valoraciones indirectas, en especial las socioeconómicas, corren paralelas a las cognitivas y afectivas o hay disonancias? A responder a estas preguntas se dedican las páginas que siguen.

\section{METODOLOGÍA}

Hemos señalado que la muestra está muy circunscrita: son estudiantes de Filología Hispánica de la Universidad de Granada, todos nacidos en la provincia de Granada, que se distribuyen como podemos observar en la tabla 1:

\begin{tabular}{|l|l|c|}
\hline \multicolumn{2}{|c|}{ GRANADA } & N \\
\hline \multirow{2}{*}{ SEXO } & Mujeres & 66 \\
\cline { 2 - 3 } & Hombres & 23 \\
\hline \multirow{2}{*}{ COLECTIVO } & Sin formación en variedades & 49 \\
\cline { 2 - 3 } & Con formación en variedades & 40 \\
\hline \multirow{2}{*}{ TOTAL } & 89 \\
\hline
\end{tabular}

Tabla 1. Características de la muestra

Observamos que tenemos dos grandes grupos: 49 estudiantes iniciales del grado, que no han tenido formación específica en variedades del español, y 40 estudiantes de los cursos finales del grado, que ya han cursado distintas materias directamente relacionadas con las variedades del español, lo que nos da un total de 89 estudiantes. Respecto al sexo de los encuestados, dada la adscripción social de la enseñanza en España, la mayoría de la muestra está constituida por mujeres, quienes casi triplican la tasa masculina.

79 encuestas se realizaron en el año 2017 y solo diez a fines del año anterior. De los encuestados, 50 eran nacidos en la capital y el resto, 39, en distintas localidades de la provincia.

Para esta investigación seguimos, como ya hemos señalado, el cuestionario del proyecto PRECAVES XXI. Para una explicación detallada del cuestionario se recomienda la consulta de Cestero Mancera y Paredes García (2018a) y Santana Marrero (2018a), autores a los que resumo en los párrafos que siguen. 
Hay dos grandes bloques de preguntas: aquellas que tienen como finalidad hacer una caracterización personal y sociogeográfica del informante y las que tienen como objeto sondear las creencias y actitudes hacia las grabaciones propuestas. El primer bloque de contenidos está conformado por preguntas en las que se piden datos del informante sobre sexo, edad, nivel de estudios, profesión, lengua materna, país de nacimiento, países hispanohablantes a los que ha viajado y hábitos en cuanto al uso de televisión y de internet.

El segundo bloque contiene doce cuestiones destinadas a conocer la opinión de los sujetos sobre las grabaciones que se les proponen. Esta parte comienza con una pregunta general abierta en la que se indaga sobre la zona en la que, en opinión del informante, se habla mejor español -si es que la hay-, y, a continuación, aparecen tres apartados a partir de los cuales se pretende obtener información acerca de los siguientes aspectos:

1) Valoración directa de la variedad: se busca saber qué juicios afectivos y cognitivos tienen los sujetos sobre las grabaciones escuchadas, qué rasgos lingüísticos les gustan más o menos y qué grado de proximidad existe entre su modalidad y la escuchada.

2) Valoración indirecta de la variedad a partir de las características personales y el estatus social atribuido al sujeto que escuchan en la grabación.

3) Valoración indirecta de la variedad a partir de la percepción que tienen los encuestados sobre esa zona o país y su cultura.

En lo que toca a las grabaciones escuchadas por los encuestados, se trata de dieciséis grabaciones, dos por cada una de las ocho variedades cultas del español propuestas por Moreno Fernández (2009, p. 79); una de ellas es una muestra de habla sin planificación previa y la otra es una muestra de un texto leído. Estas muestras se ordenan de forma aleatoria para evitar contestaciones mecánicas y todas ellas fueron grabadas por hombres y mujeres de nivel sociocultural alto de cada una de las zonas consideradas. Hubo 45 informantes que evaluaron voces masculinas y 44 que escucharon voces de mujer.

Si se tiene en cuenta que cada informante evalúa dieciséis grabaciones, obtenemos que para este estudio partimos del análisis de 1424 grabaciones.

Para el análisis de los resultados se han utilizado cálculos básicos de datos absolutos y porcentajes obtenidos directamente de las hojas de cálculo generadas por el proyecto. Asimismo, en algunas ocasiones, como se verá en el siguiente apartado, se han realizado pruebas de significación y análisis de conglomerados. Las pruebas de significación se han realizado para intentar comprobar si alguna de las variables estudiadas influye significativamente en las valoraciones subjetivas. Estas variables han sido:

a) Sexo de los encuestados (mujer u hombre)

b) Colectivo al que pertenece (sin o con formación en variedades del español)

c) Discurso evaluado (oral o leído)

d) Voz evaluada (voz de mujer o voz de hombre)

Para las pruebas de significación hemos utilizado el programa de análisis estadístico IBM SPSS 20, concretamente la determinación del $\chi 2$ de Pearson mediante tablas de contingencia y, cuando lo anterior no era posible por las características de los datos, se ha optado por la determinación de p-valor mediante la prueba de comparación de medias (ANOVA de 
un factor). El análisis de conglomerados (representado gráficamente por dendogramas) se ha efectuado con el método de medida de distancias euclideas al cuadrado.

\section{RESULTADOS}

\subsection{Jerarquía, aciertos y errores}

En Manjón-Cabeza (2018a) presentamos un análisis parcial, centrado en la autovaloración, es decir, en la percepción de la variedad andaluza y ofrecimos algunos datos generales que debemos resumir aquí, aunque ahora nos interesa la valoración de todas las variedades en conjunto.

En lo que toca al prestigio de las variedades, los encuestados se dividen entre aquellos que consideran que hay variedades mejores que otras y aquellos que consideran que no se puede señalar alguna donde se hable mejor español. Los datos aparecen en la tabla 2.

\begin{tabular}{|l|c|c|}
\hline & $\mathrm{n}$ & $\%$ \\
\hline Castilla y norte de España & 35 & 39,33 \\
\hline Andalucía & 7 & 7,87 \\
\hline América & 4 & 4,49 \\
\hline España & 3 & 3,37 \\
\hline En todas por igual & 37 & 41,57 \\
\hline No contesta & 3 & 3,37 \\
\hline Total & 89 & 100,00 \\
\hline
\end{tabular}

Tabla 2. Región o zona donde se habla mejor español

Es evidente que la variedad que los jóvenes encuestados anotan como mejor es la castellana (39,33\% del total). Las otras opciones, dentro de los que establecen jerarquías de modalidades son claramente minoritarias y solo el 7,87\% muestra el prurito localista de señalar su propia modalidad. Hay que señalar que, junto con encuestados sevillanos (Santana Marrero, 2018b), son los que evalúan peor su variedad y que le siguen, en orden ascendente de valoración de la variedad propia, los estudiantes de Santiago de Chile, Canarias, Buenos Aires, Mallorca, Medellín y Madrid; como se puede comprobar en Guerrero y San Martín (2018), Hernández y Samper (2018), Gutiérrez Böhmer y Borzi (2018), Méndez Guerrero (2018), González-Rátiva y otras (2018) y Cestero y Paredes (2018b), respectivamente.

No llega a la mitad $(41,57 \%)$, pero no es un dato desdeñable, el porcentaje que opina que no se puede señalar una variedad mejor que otra. Es, además, importante, señalar que el factor que condiciona claramente esa elección es el colectivo pre o posvariedad. Aunque hemos hecho pruebas de significación (en este caso $\chi^{2}=0,000$ ), es fácil comprobarlo porque el colectivo sin formación en materias de variedades del español, en su mayoría, opta por el establecimiento de jerarquías $(71,4 \%)$, mientras que el colectivo con formación 
en variedades la proporción se invierte y eligen la opción de igualdad de variedades en un $67,7 \%$.

En cuanto a la identificación de las variedades, hay que hacer notar que se permite la adscripción libre y, por tanto, la casuística es complicada. Hemos establecido cuatro grupos o grados en la identificación. Así, como se puede observar en la tabla 3, distinguimos los casos de adscripción exacta o acierto, como cuando, por ejemplo, se anota Cuba o Puerto Rico para una grabación de la variedad caribeña; las contestaciones erróneas, como cuando se anota Andalucía para la variedad canaria; las genéricas, sobre todo cuando se anota Latinoamérica, Hispanoamérica y similares para cualquier variedad americana, pero también cuando se optó por España para una variedad española. Por supuesto, también se anota la opción de no contestar.

\begin{tabular}{|c|c|c|c|c|c|}
\hline VARIEDAD & & $\mathrm{n} / \%$ & VARIEDAD & & $\mathrm{n} / \%$ \\
\hline \multirow{5}{*}{ CASTELLANA } & Grabaciones & 177 & \multirow{5}{*}{ ANDINA } & Grabaciones & 175 \\
\hline & Acertadas & $129 / 72,88 \%$ & & Acertadas & $43 / 24,57 \%$ \\
\hline & Erróneas & $9 / 5,08 \%$ & & Erróneas & $63 / 36,00 \%$ \\
\hline & Genéricas & $38 / 21,47 \%$ & & Genéricas & $63 / 36,00 \%$ \\
\hline & no contesta & $1 / 0,56 \%$ & & no contesta & $6 / 3,43 \%$ \\
\hline \multirow{5}{*}{ CANARIA } & Grabaciones & 173 & \multirow{5}{*}{ CHILENA } & Grabaciones & 172 \\
\hline & Acertadas & $77 / 44,51 \%$ & & Acertadas & $9 / 5,23 \%$ \\
\hline & Erróneas & $72 / 41,62 \%$ & & Erróneas & $97 / 56,40 \%$ \\
\hline & Genéricas & $14 / 8,09 \%$ & & Genéricas & $58 / 33,72 \%$ \\
\hline & no contesta & $10 / 5,78 \%$ & & no contesta & $8 / 4,65 \%$ \\
\hline \multirow{5}{*}{ MEXICANA } & Grabaciones & 175 & \multirow{5}{*}{ RIOPLATENSE } & Grabaciones & 176 \\
\hline & Acertadas & $55 / 31,43 \%$ & & Acertadas & $142 / 80,68 \%$ \\
\hline & Erróneas & $58 / 33,14 \%$ & & Erróneas & $13 / 7,39 \%$ \\
\hline & Genéricas & $53 / 30,29 \%$ & & Genéricas & $18 / 10,23 \%$ \\
\hline & no contesta & $9 / 5,14 \%$ & & no contesta & $3 / 1,70 \%$ \\
\hline \multirow{5}{*}{ CARIBEÑA } & Grabaciones & 178 & \multirow{5}{*}{ ANDALUZA } & Grabaciones & 177 \\
\hline & Acertadas & $42 / 23,60 \%$ & & Acertadas & $135 / 76,27 \%$ \\
\hline & Erróneas & $70 / 39,33 \%$ & & Erróneas & $11 / 6,21 \%$ \\
\hline & Genéricas & $60 / 33,71 \%$ & & Genéricas & $25 / 14,12 \%$ \\
\hline & no contesta & $6 / 3,37 \%$ & & no contesta & $6 / 3,39 \%$ \\
\hline
\end{tabular}

Tabla 3. Identificación de las grabaciones del conjunto de variedades. Datos absolutos y porcentajes

Se observan en la tabla 4 grandes disparidades en la identificación de variedades. Si ordenamos de mayor a menor el grado de identificación exacta, obtenemos el gráfico 1. 


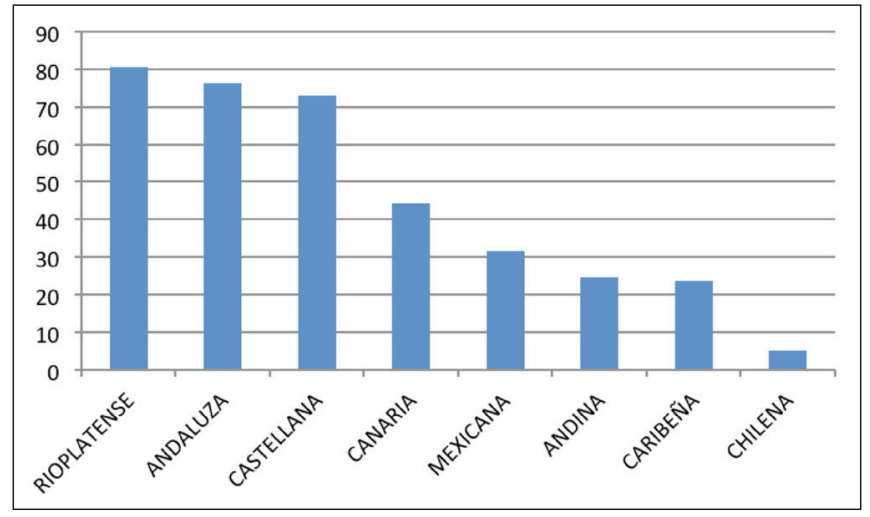

Gráfico 1. Porcentajes de identificación exacta de las variedades estudiadas

Aunque con matices, podemos afirmar que las variedades más cercanas a los encuestados, es decir, las europeas, son mejor identificadas que las americanas. Hay que resaltar que la variedad rioplatense es una excepción a esa tendencia ya que obtiene el mayor grado de reconocimiento $(80,68 \%)$, seguramente por la identificación de la pronunciación rehilada del fonema palatal. En el extremo opuesto se sitúa el grado de identificación de la variedad chilena, que solo muestra un grado de identificación del 5,23\%, dato tan exiguo que nos imposibilitará en muchos casos aplicarle pruebas estadísticas de aquí en adelante, ya que a partir de ahora solo debemos razonar con las adscripciones exactas, puesto que, si no se acierta, no podemos saber qué es lo que valora el encuestado.

\subsection{Identificación con las variedades}

La cuarta pregunta de la encuesta pide que se valore la proximidad entre la pronunciación del encuestado y la persona que ha hablado en una escala que va desde el 1 (totalmente diferente) al 6 (totalmente idéntica). Los resultados obtenidos se muestran en la tabla 4 en la que aparecen las variedades ${ }^{2}$ junto con las medias de las proximidades.

\begin{tabular}{|l|c|}
\hline Variedad & $\overline{\mathbf{X}}$ \\
\hline Andaluza & 4,39 \\
\hline Castellana & 2,98 \\
\hline Canaria & 2,90 \\
\hline Caribeña & 2,38 \\
\hline Andina & 2,00 \\
\hline Rioplatense & 1,99 \\
\hline Mexicana & 1,80 \\
\hline
\end{tabular}

Tabla 4. Promedio de la identificación de los encuestados con las grabaciones escuchadas

2 Recordemos que no razonamos con la variedad chilena. 
Está claro que la identificación se hace con la propia variedad andaluza (con un valor de 4,39). Es un promedio muy alto si consideramos que las grabaciones son de hablantes cultos sevillanos y lo esperable es que los encuestados no señalen el máximo valor ya que es muy difícil una identificación total con otro hablante, puesto que hay factores de edad o sexo, por ejemplo, que lo impiden. Creemos que la visión de la variedad andaluza donde se resalta la "extraordinaria heterogeneidad interna del andaluz, la ausencia de usos comunes" -Narbona (2009: 32) - no se puede extrapolar a las valoraciones de los propios hablantes, al menos a las de los aquí encuestados.

El resto de promedios están alejados del andaluz y se puede observar cómo estos jóvenes se identifican más con las variedades europeas (castellana y canaria) que con las americanas, alguna de las cuales se ve como muy alejada de la propia. No obstante, parece que la variedad caribeña, con 2,38 de promedio, puede actuar de nexo de unión.

Hay otro tipo de diferencias más sutiles en las actitudes de identificación con la pronunciación, puesto que en el grado de identificación con la propia variedad andaluza hay factores que han resultados significativos, mientras que esos factores casi no aparecen en las otras variedades.

La valoración del andaluz muestra fenómenos de covariación, ya que el sexo del encuestado y la voz evaluada muestran p-valores significativos. Esto se puede comprobar para la variable sexo en la tabla 5.

\begin{tabular}{|l|c|c|c|}
\hline \multicolumn{4}{|c|}{ Sexo } \\
\hline Grado de identificación & Hombres & Mujeres & \\
\hline Bajo & $6 / 17,60 \%$ & $14 / 13,90 \%$ & $20 / 14,80 \%$ \\
\hline Intermedio & $17 / 50,00 \%$ & $24 / 23,80 \%$ & $41 / 30,40 \%$ \\
\hline Alto & $11 / 32,40 \%$ & $63 / 62,40 \%$ & $74 / 54,80 \%$ \\
\hline Total & $34 / 100,00 \%$ & $101 / 100,00 \%$ & $135 / 100,00 \%$ \\
\hline$\chi 2=0,006$ & & \\
\hline
\end{tabular}

Tabla 5. Tabla de contingencia para el sexo y el grado de identificación con las grabaciones $^{3}$ andaluzas. Valores absolutos y relativos

Del examen de los datos mostrados en la Tabla 5, se puede señalar que las mujeres muestran un grado de identificación con la propia variedad más alto que el de los hombres. Eso queda patente si comparamos el porcentaje de alta identificación en las mujeres $(62,40$ $\%)$ con el de los hombres $(32,40 \%)$.

La voz evaluada también es una variable que ha resultado significativa en la valoración de la variedad propia, como se puede observar en la Tabla 6.

3 Debe observarse que el grado de identificación se ha recodificado para evitar casillas vacías en la tabla. Se ha procedido de la siguiente manera: valores 1,2 y $3=$ grado de identificación bajo; $4=$ medio; 5 y $6=$ alto. De todas formas, también se ha efectuado una comparación de medias (ANOVA de un factor) y el resultado también es significativo, pues el p-valor también fue de 0,006 . 


\begin{tabular}{|c|c|c|c|}
\hline & \multicolumn{2}{|c|}{ Voz evaluada } & Total \\
\hline Grado de identificación & Voz de hombre & Voz de mujer & \\
\hline Bajo & $14 / 18,20 \%$ & $6 / 10,30 \%$ & $20 / 14,80 \%$ \\
\hline Intermedio & $28 / 36,40 \%$ & $13 / 22,40 \%$ & $41 / 30,40 \%$ \\
\hline Alto & $35 / 45,50 \%$ & $39 / 67,20 \%$ & $74 / 54,80 \%$ \\
\hline Total & $77 / 100,00 \%$ & $58 / 100,00 \%$ & $135 / 100,00 \%$ \\
\hline
\end{tabular}

Tabla 6. Tabla de contingencia para la voz evaluada y el grado de identificación con las grabaciones andaluzas. Valores absolutos y relativos.

Quizá debido a que las mujeres conforman la mayoría de la muestra hay una identificación mayor con la voz de mujer que con la de hombre, como resulta evidente si confrontamos el porcentaje de alta identificación para la variedad andaluza en el caso de la voz femenina $(67,20 \%)$ frente a los más de veinte puntos menos de identificación con la voz masculina $(45,50 \%)$.

Es muy revelador que, para las otras variedades hispánicas, tras las pruebas efectuadas, no hayamos encontrado fenómenos de covariación en las elecciones de los encuestados, lo que da idea de la homogeneidad del grado de identificación ${ }^{4}$ con las variedades no propias. Una excepción la constituye la voz evaluada (de hombre o de mujer) que sí ha resultado significativa en las valoraciones de la variedad rioplatense, como queda de manifiesto en la Tabla 7.

\begin{tabular}{|l|c|c|c|}
\hline \multicolumn{4}{c|}{ Voz evaluada } \\
\hline Grado de identificación & Voz de hombre & Voz de mujer & \\
\hline Bajo & $23 / 34,30 \%$ & $32 / 45,10 \%$ & $55 / 39,90 \%$ \\
\hline Intermedio & $18 / 26,90 \%$ & $26 / 36,60 \%$ & $44 / 31,90 \%$ \\
\hline Alto & $26 / 38,80 \%$ & $13 / 18,30 \%$ & $39 / 28,30 \%$ \\
\hline Total & $67 / 100,00 \%$ & $71 / 100,00 \%$ & $138 / 100,00 \%$ \\
\hline$\chi^{2}=0,028$ & & \\
\hline
\end{tabular}

Tabla 7. Tabla de contingencia para la voz evaluada y el grado de identificación con las grabaciones rioplatenses. Valores absolutos y relativos.

4 Como el grado de identificación con las variedades no propias es mucho menor (obsérvese la Tabla 4) y, por tanto, los valores 4, 5 y 6 de la encuesta no contaban con muchas elecciones, la recodificación para todas la variedades no andaluzas se hizo de la siguiente manera: valor $1=$ grado de identificación bajo, valor $2=$ grado de identificación intermedia y el resto de valores = grado de identificación alta. 
Los datos de la tabla 7 nos sugieren un grado de identificación menor con las grabaciones femeninas. Si trasladamos los porcentajes a un gráfico obtenemos el gráfico 2, donde se puede apreciar mejor.

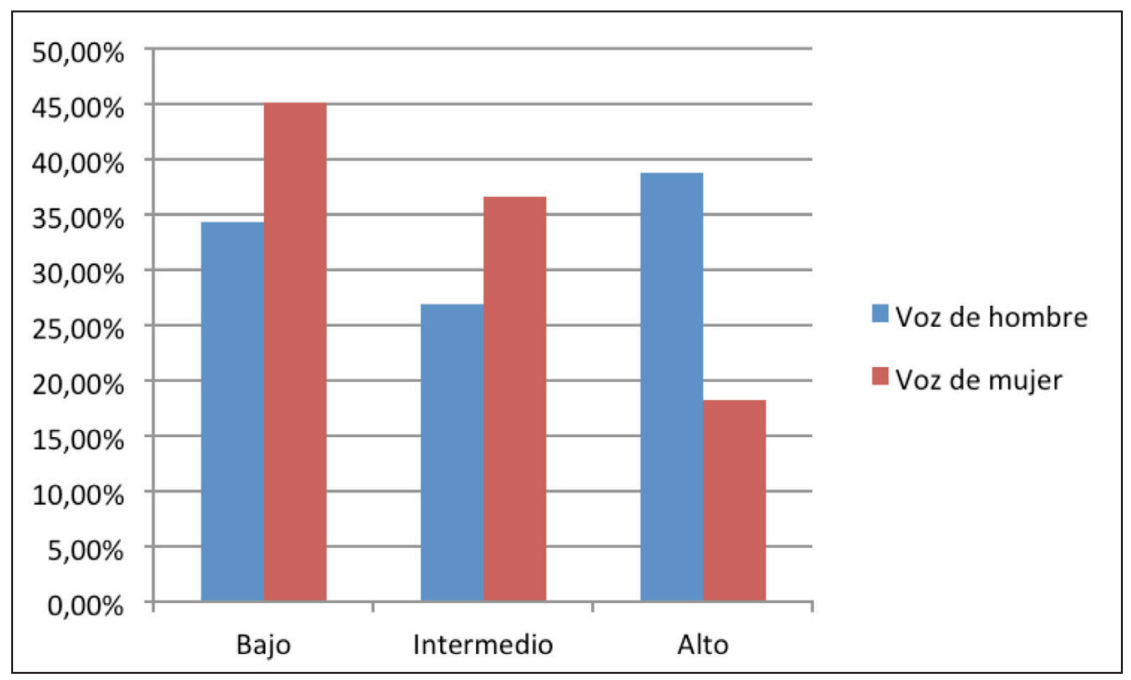

Gráfico 2. Porcentajes del grado de identificación con la variedad rioplatense según la voz evaluada

Del gráfico 2 debemos resaltar que las valoraciones de la voz femenina están claramente escoradas hacia las valoraciones bajas (izquierda del gráfico), frente a las valoraciones de la voz masculina que muestran una homogeneidad mayor. Es posible que ese alejamiento actitudinal de la voz femenina rioplatense, más llamativo si se tiene en cuenta que la muestra encuestada es mayoritariamente de mujeres, seguramente tiene que ver la realidad sociolingüística de la variedad americana considerada. Es conocido que uno de los rasgos más identificadores de la variedad rioplatense, frente a otras variedades del español, es el tratamiento de los sonidos palatales. Los nuevos sonidos palatales, especialmente el rehilamiento de $/ \mathrm{j} /$ en $/ 3 /$ y su posterior ensordecimiento en $/ \mathrm{J} /$, son patrocinados por las mujeres, como ya indicó el trabajo pionero de Fontanella de Weinberg (1979); de modo que podemos plantear la hipótesis de que el ensordecimiento palatal actúa como un rasgo que provoca una valoración de lejanía. Si estos sonidos palatales son más patentes en las mujeres rioplatenses, se puede explicar las valoraciones mostradas en la Tabla 7 y en el Gráfico 2.

\subsection{Valoraciones directas}

La encuesta de PRECAVES XXI plantea la valoración en una escala del 1 al 6 de once características de la voz escuchada; cinco de ellas podemos calificarlas de cognitivas (áspera/suave, monótona/variada, rural/urbana, lenta/rápida, confusa/clara) y seis, de afectivas (desagradable/agradable, complicada/ sencilla, distante/cercana, dura/blanda, aburrida/divertida, fea/bonita). 
Las medias ${ }^{5}$ de estas valoraciones directas se muestran en la Tabla 8, en la que también ofrecemos las grabaciones identificadas con exactitud (n), que son las que sirven de base para el análisis.

\begin{tabular}{|l|r|r|r|r|}
\hline VARIEDAD & $\mathrm{n}$ & Media cognitiva & Media afectiva & \multicolumn{1}{c|}{ Media general } \\
\hline CASTELLANA & 129 & 4,00 & 3,61 & 3,80 \\
\hline ANDALUZA & 135 & 3,60 & 4,02 & 3,81 \\
\hline CANARIA & 77 & 3,90 & 4,08 & 3,99 \\
\hline MEXICANA & 56 & 3,80 & 4,43 & 4,11 \\
\hline CARIBENA & 42 & 3,90 & 4,43 & 4,17 \\
\hline ANDINA & 43 & 3,70 & 3,80 & 3,75 \\
\hline RIOPLATENSE & 142 & 3,80 & 3,98 & 3,89 \\
\hline
\end{tabular}

Tabla 8. Medias de las valoraciones directas de las variedades

De los datos presentados en la tabla 8 se puede observar que todas las variedades tienden a tener una valoración global positiva, aunque hay diferencia entre las variedades mejor valoradas globalmente, que son la caribeña y la mexicana, y las peores valoradas, que son las variedades andinas y la castellana (esta última casi empatada con la valoración de la variedad propia).

Estos datos conviene pormenorizarlos ya que, como también se puede deducir de la tabla 8 , no son coincidentes las valoraciones cognitiva y las afectivas. Es cierto que las variedades caribeña y mexicana son las que obtienen mejor valoración afectiva, pero son sobrepasadas en la valoración cognitiva por la castellana, que ocupa los dos extremos: es la mejor valorada cognitivamente $(4,00)$ pero la peor valorada afectivamente $(3,61)$. Estos datos son coincidentes con los de los estudiantes sevillanos analizados en Santana (2018: 82).

Si seguimos pormenorizando podemos ver el comportamiento de las variedades en lo que toca a las características concretas. Para ello hemos elaborado dos tablas (Tablas 9 y 10). En la tabla 9 mostramos las medias de las valoraciones directas de carácter cognitivo.

\begin{tabular}{|l|r|r|r|r|r|}
\hline & \multicolumn{1}{|c|}{ Áspera } & \multicolumn{1}{c|}{ Monótona } & \multicolumn{1}{c|}{ Rural } & \multicolumn{1}{c|}{ Lenta } & \multicolumn{1}{c|}{ Confusa } \\
\hline VARIEDAD & & & & & 1,67 \\
\hline Castellana & 3,46 & 4,13 & 1,92 & 3,75 & 2,91 \\
\hline Andaluza & 3,76 & 3,37 & 3,69 & 3,24 & 2,81 \\
\hline Canaria & 3,17 & 3,22 & 3,03 & 3,25 & 2,54 \\
\hline Mexicana & 2,30 & 3,45 & 3,42 & 4,32 & \\
\hline
\end{tabular}

5 Para el análisis de los datos de las valoraciones directas ha de tenerse en cuenta que la escala va del 1 al 6 , por lo que el valor intermedio es 3,5. Asimismo, debe hacerse notar que en la tabla 8 las medias se han calculado siempre teniendo en cuenta el polo "positivo" de la dicotomía. Por ejemplo, la variedad castellana es valorada con 1,92 para la característica rural, de modo que, en este caso, se tiene en cuenta para hacer la media cognitiva el valor 4,08 (desplazado a la urbanidad). 


\begin{tabular}{|l|r|r|r|r|r|}
\hline & \multicolumn{1}{|c|}{ Áspera } & \multicolumn{1}{c|}{ Monótona } & \multicolumn{1}{c|}{ Rural } & \multicolumn{1}{c|}{ Lenta } & \multicolumn{1}{c|}{ Confusa } \\
\hline VARIEDAD & & & & & \\
\hline Caribeña & 2,40 & 3,19 & 3,17 & 4,02 & 2,52 \\
\hline Andina & 3,28 & 3,49 & 3,14 & 3,67 & 2,86 \\
\hline Rioplatense & 3,17 & 3,40 & 2,81 & 3,53 & 2,82 \\
\hline
\end{tabular}

Tabla 9. Media de las valoraciones directas de características cognitivas

Si analizamos los datos de la tabla 9 estamos en condiciones de explicar las medias generales ofrecidas en la tabla 8. Así, observamos que el mayor grado de aspereza es atribuido a dos variedades europeas: la propia y la castellana, mientras que la suavidad caracteriza a dos variedades americanas: la caribeña y la mexicana. La monotonía, para estos futuros profesores, es claramente una característica del español de Castilla, que, por el contrario, es evaluado muy positivamente por su claridad. La lentitud es atribuida a las variedades caribeña y mexicana que, recordemos, se caracterizan también por su suavidad. No obstante, en estas evaluaciones, queremos destacar la atribución de carácter rural o urbano, porque, como ha sido señalado -por ejemplo, en Garvin y Mathiot (1974: 303-304)- la lengua estándar está directamente asociada a la urbanización, de modo que estos datos nos pueden dar pistas fiables sobre el prestigio (o la falta de este) asociado a las distintas variedades. Para ilustrar este punto ofrecemos el gráfico 3 en el que ordenamos las variedades evaluadas según su ruralidad.

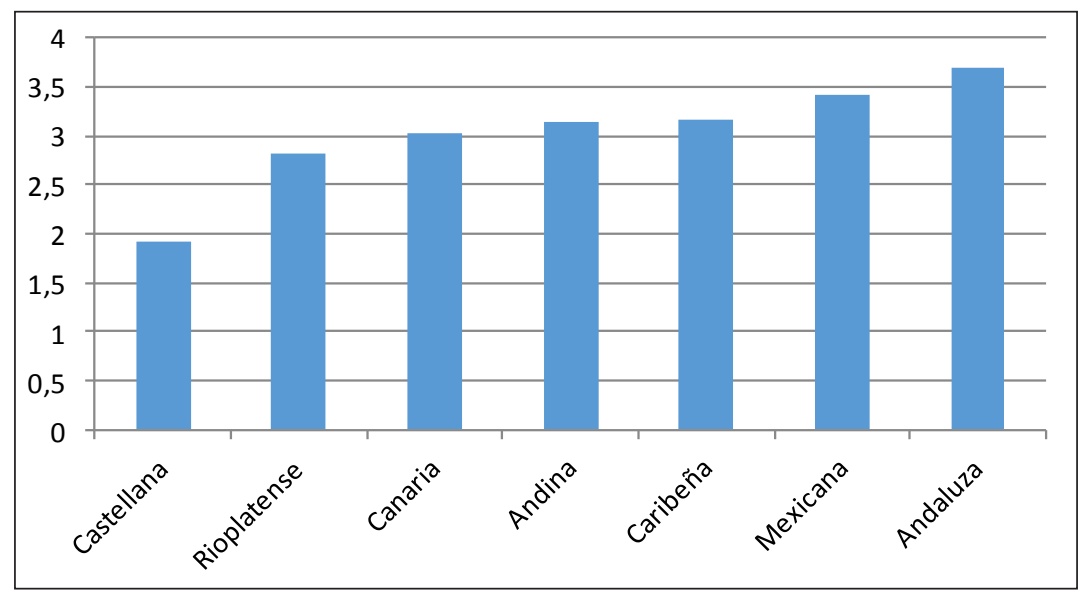

Gráfico 3. Valoración de la ruralidad para las variedades del español

En el gráfico 3 queda patente que hay una variedad claramente identificada como urbana: la variedad castellana. Si tenemos en cuenta que el punto intermedio de la escala es 3,5 todas las demás variedades quedan englobadas en la urbanidad, excepto una: la variedad propia, la andaluza. Estamos ante la presencia de un tópico muy arraigado en el imaginario de los hablantes del español de Europa. Además, es un tópico fácilmente refutable, ya que 
según datos del censo del INE de 2018, tan solo el 19,3\% de la población andaluza vivía en municipios de menos de 10000 habitantes, por debajo de la media nacional $(20,6 \%)$, mientras que en Castilla y León lo hace el 43,8 \%.

En la Tabla 10 mostramos las medias de las valoraciones directas de carácter afectivo.

\begin{tabular}{|l|r|r|r|r|r|r|}
\hline & Agradable & \multicolumn{1}{c|}{ Sencilla } & \multicolumn{1}{c|}{ Cercana } & Blanda & \multicolumn{1}{c|}{ Divertida } & \multicolumn{1}{c|}{ Bonita } \\
\hline VARIEDAD & & & & & & \\
\hline Castellana & 4,22 & 4,53 & 3,38 & 3,52 & 2,39 & 3,71 \\
\hline Andaluza & 4,23 & 4,24 & 4,59 & 3,59 & 3,48 & 3,97 \\
\hline Canaria & 4,16 & 3,97 & 4,24 & 3,99 & 3,81 & 4,28 \\
\hline Mexicana & 4,54 & 4,36 & 4,59 & 4,43 & 4,09 & 4,57 \\
\hline Caribeña & 4,64 & 4,24 & 4,80 & 4,45 & 3,95 & 4,50 \\
\hline Andina & 3,81 & 4,12 & 4,14 & 3,67 & 3,14 & 3,93 \\
\hline Rioplatense & 4,25 & 4,02 & 3,92 & 3,87 & 3,68 & 4,24 \\
\hline
\end{tabular}

Tabla 10. Media de las valoraciones directas de características afectivas

Ahora, según se desprende de los datos de la tabla 10, las valoraciones cambian con respecto a las características cognitivas. Observamos que la variedad castellana que era muy bien valorada cognitivamente, ahora es la peor valorada respecto a la cercanía, la dureza y el carácter divertido. También parece evidente que las variedades mejor valoradas afectivamente son la mexicana y caribeña, que alcanza los mejores resultados en lo que toca a agrado, sencillez, cercanía (por encima de la propia variedad), blandura, diversión y belleza.

Queda por responder a la pregunta de si hay alguna variable que incida significativamente en las valoraciones directas. En este caso hemos efectuado análisis ANOVA y tabulaciones cruzadas solo para las medias de las valoraciones de las características cognitivas y afectivas, que mostrábamos en la tabla 8 . Las pruebas estadísticas nos indican que la incidencia de las variables consideradas es escasa en estas valoraciones directas. Solo interviene la voz evaluada (de hombre o de mujer) y no en todas las ocasiones ni en la misma dirección. De este modo, podemos señalar lo siguiente:

a) La valoración afectiva de la variedad castellana está influida significativamente $\left(\chi^{2}\right.$ de Pearson $=0,037)$ porque es mejor cuando la voz es de hombre. Podemos comprobar este punto porque la media de las evaluaciones afectivas de las voces masculinas es de 3,8, mientras que esa media baja a 3,4 cuando se trata de una voz femenina.

b) En el caso de la variedad canaria, también actúa de manera significativa la variable de la voz evaluada para la valoración afectiva $\left(\chi^{2}\right.$ de Pearson= 0.001$)$, pero, al contrario de lo que ocurre en la castellana, ahora es mejor evaluada la voz de mujer. De hecho, hay una diferencia importante entre la media de las valoraciones afectivas en caso de voz masculina $(3,8)$ y la correspondiente a la voz de mujer, que sube hasta 4,4 .

c) La variedad andina es la única a la que la voz evaluada afecta significativamente tanto a la valoración de las características cognitivas $\left(\chi^{2}=0,004\right)$ como a la valoración de las características afectivas $\left(\chi^{2}=0,001\right)$. En ambos casos son mejor evaluadas las 
audiciones de las voces de hombre, con unas diferencias de medias bastante altas, puesto que, para la valoración cognitiva, se pasa de una media de 4,1 para la voz masculina a 3,3 para la voz femenina. Para la valoración de las características afectivas se pasa de 4,2 de la voz masculina a 3,3 de las voces de mujer.

d) La variedad rioplatense coincide con la canaria en que para ella la voz evaluada incide significativamente en la valoración de las características afectivas $\left(\chi^{2}=0.001\right)$ ya que es mejor evaluada la voz de mujer, con una media de 4,2, que la de hombre, que baja a 3,7 .

Para intentar dar una visión global de cómo valoran las distintas variedades los futuros profesores de español granadinos, hemos efectuado un análisis de conglomerados jerárquico con el método del cálculo de la distancia euclidea al cuadrado. El resultado queda plasmado en el dendograma que aparece en el Gráfico 4.

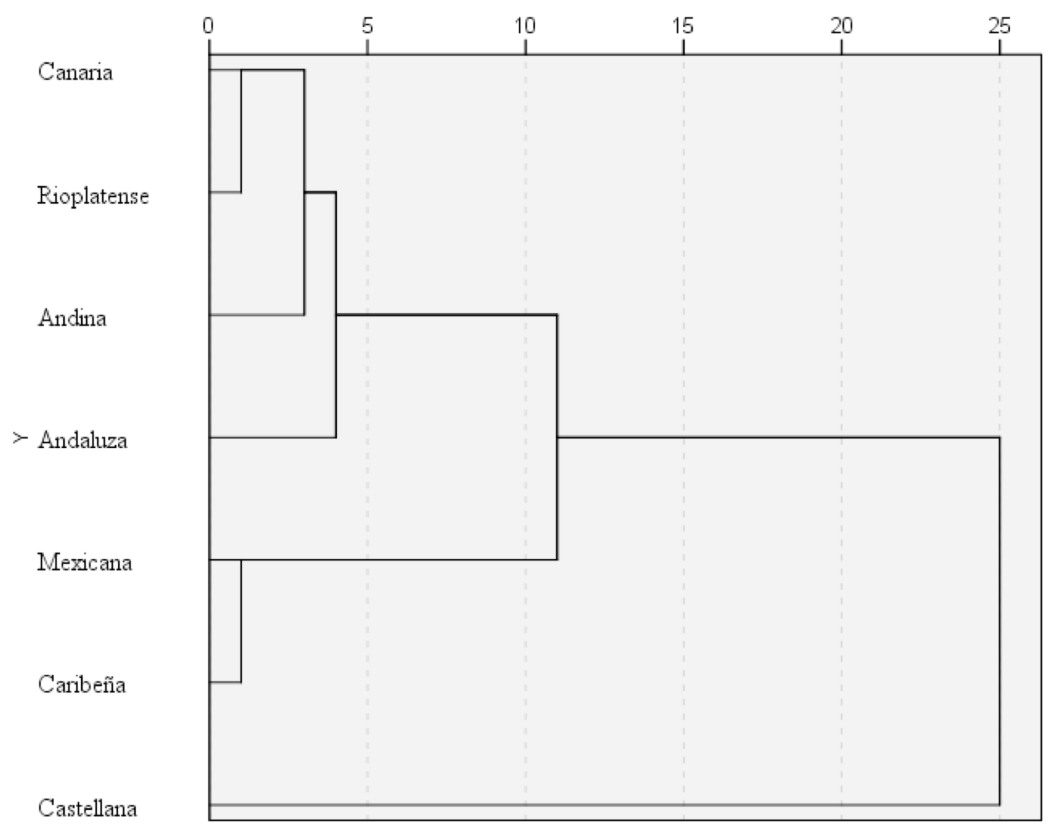

Gráfico 4. Dendrograma de distancias para las valoraciones directas de las variedades

Para la correcta interpretación del dendrograma del Gráfico 4 ha de tenerse en cuenta que hemos partido de las medias de las valoraciones directas, tanto cognitivas como afectivas, es decir, de los datos que aparecen en las tablas 9 y 10. En el gráfico 4 podemos observar que hay dos conglomerados formados por dos variedades con valoraciones muy similares: por una lado, las variedades canaria y rioplatense y, por otro, las variedades mexicana y caribeña; aunque estos dos conglomerados no están muy próximos entre sí, seguramente porque las variedades mexicana y caribeña se diferencian de las demás en la alta valoración afectiva. 
Parece también evidente que la valoración de la variedad castellana tiene unos valores muy distintos de todas las demás puesto que solo se une al resto como último conglomerado. Este gráfico no hace más que reflejar que, para los encuestados, la variedad castellana tiene una valoración muy diferente de las demás, puesto que obtiene muy altos valores para muchas características cognitivas y muy bajos para muchas características afectivas.

\subsection{Valoraciones indirectas}

La última parte de la encuesta de PRECAVES XXI se centra en las valoraciones indirectas. Se pide a los informantes que den su opinión sobre la persona que habla, puesto que, en realidad, valoran la variedad que oyen, que asocian a determinados rasgos. En esta parte del cuestionario, se indaga en la valoración socioeconómica del emisor, las características personales del hablante, y la valoración de la zona o país y su cultura.

Para la valoración socioeconómica se utilizan preguntas cerradas que piden la opinión del informante acerca del puesto de trabajo (poco, bien o altamente cualificado), del nivel de ingresos (bajos, medios o altos) y del nivel de estudios (sin estudios, primarios, secundarios, universitarios) que considera que tiene la persona que está hablando.

Los resultados porcentuales de la valoración socioeconómica se muestran en la tabla 11.

\begin{tabular}{|l|l|r|r|r|r|r|r|r|}
\hline \multicolumn{2}{|c|}{} & $\begin{array}{c}1 \\
(\mathrm{n}=135)\end{array}$ & $\begin{array}{c}2 \\
(\mathrm{n}=129)\end{array}$ & $\begin{array}{c}3 \\
(\mathrm{n}=77)\end{array}$ & $\begin{array}{c}4 \\
(\mathrm{n}=55)\end{array}$ & $\begin{array}{c}5 \\
(\mathrm{n}=42)\end{array}$ & $\begin{array}{c}6 \\
(\mathrm{n}=43)\end{array}$ & $\begin{array}{c}7 \\
(\mathrm{n}=142)\end{array}$ \\
\hline \multirow{5}{*}{ Trabajo } & No contesta & 0,74 & 0,78 & 1,30 & 1,82 & 0,00 & 0,00 & 1,41 \\
\cline { 2 - 10 } & Poco cualificado & 24,44 & 3,88 & 9,09 & 36,36 & 9,52 & 25,58 & 6,34 \\
\cline { 2 - 10 } & Bien cualificado & 68,15 & 54,26 & 76,62 & 58,18 & 83,33 & 62,79 & 81,69 \\
\cline { 2 - 9 } & $\begin{array}{l}\text { Altamente } \\
\text { cualificado }\end{array}$ & 6,67 & 41,09 & 12,99 & 5,45 & 7,14 & 11,63 & 10,56 \\
\hline \multirow{5}{*}{ Ingresos } & No contesta & 0,74 & 0,78 & 0,00 & 1,82 & 0,00 & 0,00 & 0,70 \\
\cline { 2 - 10 } & Bajos & 13,33 & 0,78 & 7,79 & 27,27 & 7,14 & 20,93 & 7,04 \\
\cline { 2 - 10 } & Medios & 77,78 & 58,91 & 74,03 & 63,64 & 76,19 & 62,79 & 82,39 \\
\cline { 2 - 9 } & Altos & 8,15 & 39,53 & 18,18 & 7,27 & 16,67 & 13,95 & 9,86 \\
\hline \multirow{5}{*}{ Estudios } & No contesta & 0,74 & 0,78 & 2,60 & 1,82 & 0,00 & 0,00 & 1,41 \\
\cline { 2 - 9 } & Sin estudios & 2,96 & 0,00 & 0,00 & 3,64 & 2,38 & 4,65 & 0,70 \\
\cline { 2 - 9 } & Primarios & 20,00 & 3,88 & 5,19 & 30,91 & 7,14 & 20,93 & 8,45 \\
\cline { 2 - 9 } & Secundarios & 51,85 & 24,81 & 46,75 & 45,45 & 42,86 & 23,26 & 51,41 \\
\cline { 2 - 9 } & Universitarios & 24,44 & 70,54 & 45,45 & 18,18 & 47,62 & 51,16 & 38,03 \\
\hline
\end{tabular}

Tabla 11. Porcentajes de valoración socioeconómica. $1=$ andaluz, $2=$ castellano, $3=$ canario, 4=mexicano-centroamericano, $5=$ caribeño, $6=$ andino, $7=$ rioplatense

Son bastantes datos, pero podemos intentar resumir en que hay una variedad muy bien valorada en los tres aspectos: la castellana; un grupo intermedio formado por las variedades canaria, caribeña y rioplatense y un grupo relativamente mal valorado en el que están las 
variedades mexicana, andina y andaluza. Podemos aducir algunos datos de la tabla 11 a modo de ejemplo. Obsérvese, en el caso de la cualificación baja del trabajo, que las variedades andaluza, mexicana y caribeña arrojan porcentajes de más del $20 \%$, mientras que las demás bajan todas del $10 \%$ y una de ellas, la castellana, tiene una adscripción a la baja cualificación de solo el 3,88\%.

Algo parecido sucede con la valoración del nivel de ingresos. La adscripción al nivel de ingresos bajos muestra valores muy parecidos para las variedades canaria, caribeña y rioplatense (alrededor de un $7 \%$ ), casi inexistentes en el caso de la variedad castellana, mientras que en la andaluza suben al $13.33 \%$, porcentaje ampliamente superado por las variedades andina $(20,93 \%)$ y mexicano-centromericana $(27,77 \%)$.

Se vuelven a hacer adscripciones equiparables a las anteriores en el caso del nivel de estudios. Si nos fijamos en la fila de los porcentajes de adscritos a los estudios primarios solo las variedades andaluza, mexicana y andina superan el $20 \%$. Eso contrasta con el elevadísimo porcentaje de adscripción a los estudios universitarios de los hablantes de la variedad castellana, con un $70,54 \%$.

Si todos los porcentajes presentados en la tabla 11 los trasladamos a un análisis de conglomerados podemos ver los grupos creados en el Gráfico 5.

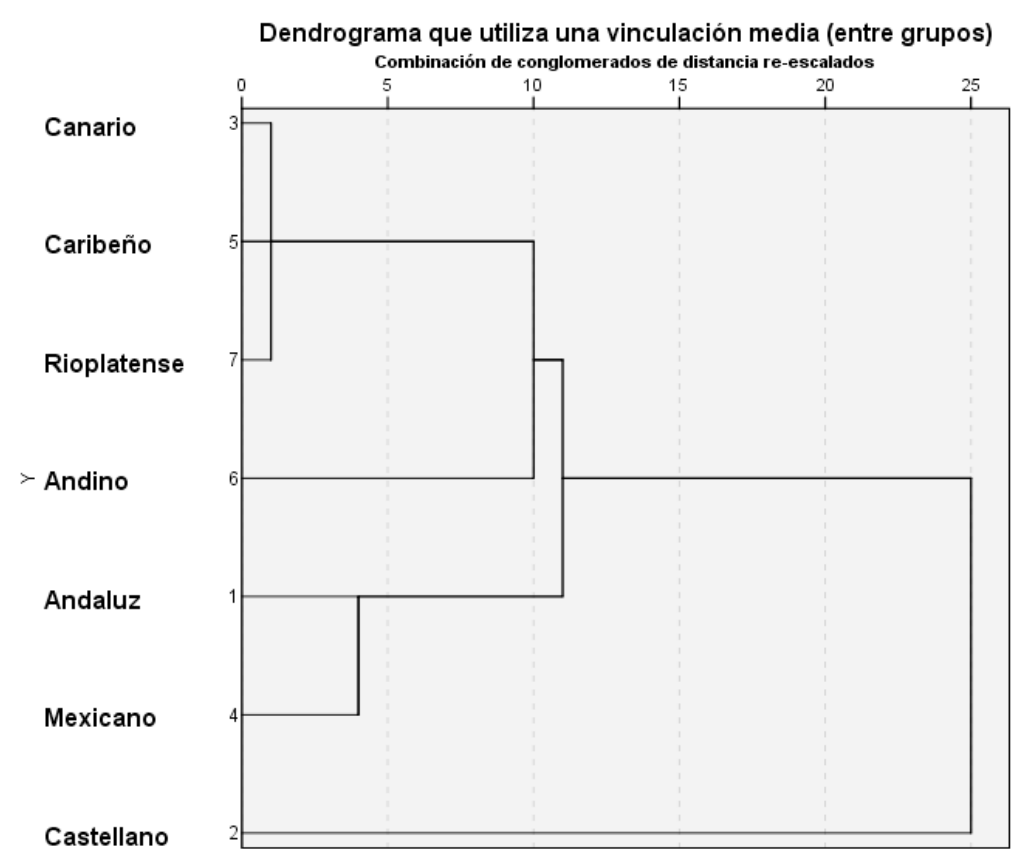

Gráfico 5. Dendrograma de distancias para las valoraciones socioeconómicas de las variedades

En el gráfico 5 podemos observar el grupo o cluster de las variedades canaria, caribeña y rioplatense que son muy homogéneas entre sí. También es parecida la valoración global 
del grupo formado por la variedad andaluza y mexicana, a la que se suma, a algo más de distancia, la variedad andina. Otra vez queda muy lejos de la valoración de estas variedades la castellana, altamente valorada en todos los aspectos.

Si bien podemos explicarnos la mala valoración de la variedad propia por la estigmatización histórica, no es tan fácil esclarecer la mala valoración socioeconómica de las variedades mexicana y andina. Hay que tener en cuenta que son variedades con muchas similitudes lingüísticas, que, de hecho, los encuestados confunden con frecuencia; pero, en realidad, en Granada solo están en contacto significativo con hablantes de una de ellas: la andina. Es más que posible que en esa mala valoración económica influya la percepción negativa que se tiene de los nuevos granadinos de origen ecuatoriano, comunidad con una fuerte presencia en Granada - Manjón-Cabeza (2018b, p. 68)- (y en toda España).

La siguiente valoración indirecta consiste en una pregunta de escala de diferencial semántico, en una gradación de 1 a 6 , donde se valoran las características personales de la persona que habla: inteligencia, simpatía, cercanía, erudición y educación.

En la Tabla 12 se muestran los resultados de las valoraciones de las personas.

\begin{tabular}{|l|r|r|r|r|r|}
\hline & Inteligente & \multicolumn{1}{|c|}{ Simpática } & \multicolumn{1}{c|}{ Cercana } & \multicolumn{1}{c|}{ Culta } & \multicolumn{1}{c|}{ Educada } \\
\hline Andaluza & 4,31 & 4,52 & 4,56 & 4,07 & 4,80 \\
\hline Castellana & 4,90 & 3,46 & 3,39 & 4,81 & 4,99 \\
\hline Canaria & 4,59 & 4,89 & 4,75 & 4,50 & 5,07 \\
\hline Mexicana & 4,07 & 4,98 & 4,79 & 4,02 & 4,95 \\
\hline Caribeña & 4,62 & 4,88 & 4,93 & 4,19 & 5,10 \\
\hline Andina & 4,08 & 3,80 & 4,05 & 3,88 & 4,48 \\
\hline Rioplatense & 4,48 & 4,24 & 4,21 & 4,36 & 4,77 \\
\hline
\end{tabular}

Tabla 12. Medias de las valoraciones indirectas de características personales

Respecto a la tabla 12 se pueden comentar varios aspectos principales. El primero es que los futuros profesores tienden a valorar positivamente a todas las personas que han escuchado: muy pocos valores están por debajo de 3,5, valor que marca la divisoria de la escala de 1 a 6 . Solo hay dos valores negativos, puesto que los encuestados valoran negativamente la simpatía $(3,46)$ y la cercanía $(3,39)$ de los hablantes castellanos, mientras que son muy bien valorados en educación, y, sobre todo, en inteligencia y cultura donde alcanzan las puntuaciones más altas. No podemos dejar de anotar que los futuros profesores consideran más cercanas zonas como la rioplatense, caribeña o mexicano-centroamericana que la castellana. La falta de afectividad hacia la zona con la que se identifica la variedad castellana se hace patente.

Los datos nos arrojan, dentro de la general consideración positiva, matices interesantes puesto que la variedad andina no queda del todo bien parada puesto que arroja valores relativamente bajos en simpatía y en cultura. Otra vez sospechamos que hay algún componente xenófobo que influye en esos resultados. Debe observarse que, en contra de lo que sucedía en el apartado de las valoraciones económicas, ahora la variedad mexicano-centroamericana no sigue las pautas de la andina. 
En el lado contrario, se vuelven a ponderar las variedades caribeña y canaria, especialmente en lo que toca a educación, con valores que sobrepasan el 5.

En lo que toca a la valoración indirecta del país o zona de procedencia del hablante, también se hace mediante una escala de diferencial semántico del 1 al 6 donde se valoran los siguientes aspectos: retrasado/avanzado, aburrido/divertido, extraño/familiar, feo/bonito.

En la tabla 13 ofrecemos las medias de las valoraciones indirectas de la zona o país.

\begin{tabular}{|l|r|r|r|r|}
\hline & \multicolumn{1}{|l|}{ Avanzado } & \multicolumn{1}{l|}{ Divertido } & \multicolumn{1}{l|}{ Familiar } & \multicolumn{1}{l|}{ Bonito } \\
\hline Andaluza & 4,44 & 4,96 & 5,24 & 5,27 \\
\hline Castellana & 4,84 & 3,90 & 3,96 & 4,73 \\
\hline Canaria & 4,42 & 5,13 & 4,92 & 5,45 \\
\hline Mexicana & 3,57 & 4,59 & 4,23 & 5,04 \\
\hline Caribeña & 3,40 & 4,39 & 4,55 & 5,07 \\
\hline Andina & 3,02 & 3,81 & 3,90 & 4,43 \\
\hline Rioplatense & 4,12 & 4,53 & 4,32 & 5,10 \\
\hline
\end{tabular}

Tabla 13. Medias de las valoraciones indirectas del país o zona

Otra vez, si consideramos los datos de la tabla 13, observamos que hay altas valoraciones generales, aunque no deja de haber diferencias importantes en las valoraciones. Creemos que en esta ocasión actúa el prurito localista en los futuros profesores porque ahora la variedad mejor puntuada es la andaluza -junto con la canaria-, sobre todo porque es considerada, como es lógico, muy familiar. Llama la atención la menor consideración, en general, del carácter avanzado de los países americanos respecto de las zonas europeas y la disociación respecto a la zona norteña de España que se considera la más avanzada $(4,84)$, pero de las menos divertidas, familiares o bonitas.

Otra vez la zona peor evaluada, con diferencia, es la andina ya que arroja los datos más bajos en todos los aspectos valorados en este apartado. No deja de sorprender esta relativa mala valoración de una zona que comprende desde Colombia hasta Bolivia y no podemos dejar de sospechar el componente psico-social ligado a la emigración desde alguno de estos países, sobre todo la ecuatoriana.

\section{CONCLUSIONES}

El español es una lengua con una gran extensión geográfica y, por tanto, policéntrica. Las distintas variedades del español son objeto de valoraciones por sus hablantes. En el caso de los futuros profesores de español granadinos las actitudes y creencias hacia estas variedades tendrán una repercusión directa en su quehacer profesional.

Durante mucho tiempo la visión jerarquizada de las variedades estuvo avalada por las autoridades académicas, pero hoy en día, al menos oficialmente, la política lingüística oficial es otra. Sin embargo, observamos como los estudiantes recién ingresados en el grado de Filología Hispánica siguen teniendo una visión jerárquica donde la variedad del centro y norte 
de la península ibérica es considerada más correcta que el resto. Esta visión se atenúa con los años. De este modo, en los cursos superiores, la consideración tiende a ser más igualitaria.

La identificación de las distintas variedades se puede considerar alta si tenemos en cuenta la adscripción genérica, es decir, la distinción general entre variedades europeas y variedades americanas. Esa identificación es media, incluso baja, se consideramos las adscripciones exactas. En ese caso son bien reconocidas las variedades andaluza, castellana y rioplatense. El conocimiento de las dos primeras se explica porque una es la variedad propia, con la que se identifican, y otra es muy cercana, lógicamente presente en sus vidas cotidianas. El alto reconocimiento de la variedad rioplatense se puede deber a la saliencia fonética de sus característicos sonidos palatales, y a la relativa familiaridad cotidiana de estos futuros profesores con personajes rioplatenses de relevancia mediática, sobre todo en el ámbito del deporte. Hay que destacar, con cierta preocupación, el relativamente bajo grado de reconocimiento exacto de la variedad canaria y el muy escaso de la variedad chilena.

Las valoraciones directas tienen vertientes cognitivas y afectivas. En la cognitiva es evidente que es la variedad castellana la mejor valorada, mientras que la peor valorada es la propia, lo que refleja unas situación psicosocial del andaluz muy negativa para los propios futuros profesores andaluces. En estas valoraciones tiene especial peso la consideración de variedad urbana para la castellana (en menor medida la rioplatense): la variedad del norte y centro español se identifica con Madrid, mientras que la propia es considerada rural, cuando sabemos que no es así. Quizá una larga tradición de estudios etnográficos rurales influye en esta visión. También es llamativo que haya una división entre una variedad clara (castellana) y las demás, más confusas, en especial la andaluza. Creemos que aquí actúa el repetido y absurdo mito de que los andaluces "se comen" las letras, con una confusión entre oralidad y escritura que sigue perviviendo a pesar de sus estudios.

En las valoraciones directas de carácter afectivo se vuelven las tornas: la variedad castellana es la peor considerada, pero no es la andaluza, contrariamente a lo esperado, la mejor evaluada, sino algunas americanas, como la caribeña y la mexicana. No consideramos positiva la mala valoración afectiva de la variedad castellana, tan cercana a estos futuros profesores, pero creemos que está reflejando un rechazo inconsciente al estándar en buena medida impuesto. Cuestión aparte es la mala valoración general de la variedad andina. Nos gustaría equivocarnos pero suponemos que la explicación tiene que ver con discriminación -seguramente inconsciente- hacia poblaciones inmigrantes.

Estas valoraciones directas están en consonancia con las indirectas, en especial con la adscripción socioeconómica de los hablantes: la variedad mejor considerada cognitivamente es la que se asocia al nivel más alto de estudios, de ingresos y de cualificación laboral. Las valoraciones indirectas que tienen en cuenta a las personas o la zona o país también nos ofrecen datos que tienen paralelo en las valoraciones directas. Vuelve a llamar la atención la relativa mala valoración de los países andinos (y sus hablantes, por tanto) que solo podemos explicar como reflejo de algún tipo de rechazo xenofóbico subyacente hacia la emigración.

Creemos, para finalizar, que las actitudes y creencias son un capítulo crucial en la formación del profesorado de lenguas. Es difícil erradicar viejas creencias que tienden a ponderar unas variedades en detrimento de otras. Esto es especialmente relevante en el caso de los futuros profesores andaluces que se debaten entre ideas muy asentadas en la tradición, tanto popular como científica, que asocia su variedad con ruralidad, atraso, falta de estudios, ámbitos cómicos de los medios de comunicación, etc. No obstante, lo verdaderamente grave 
es no tener conciencia de que estas actitudes y creencias son subjetivas, sin apoyo científico alguno y, lo peor, que nos pueden llevar a discriminar a personas y colectivos solo por una forma de hablar que no coincida con la nuestra.

\section{REFERENCIAS BIBLIOGRÁFICAS}

Alcaraz Masats, F. et al. (1979). El andaluz. Informe sobre la conciencia lingüística en Andalucía. Jaén: Dpto. de Lingüística y Crítica Literararia. Colegio Universitario de Jaén.

Almeida, M. (2003). Sociolingüistica, $2^{\mathrm{a}}$ ed. Corregida y aumentada, La Laguna: Univ. de La Laguna.

Carbonero Cano, P. (2003). "Norma estándar y actitud sociolingüística", en Carbonero Cano (Ed.), Estudios de sociolingüística andaluza. Sevilla: Secretariado de Publicaciones de la Universidad de Sevilla, 21-29.

Cestero Mancera, A. M y Paredes García, F. (2015). "Creencias y actitudes hacia las variedades normativas del español actual: primeros resultados del Proyecto PRECAVES-XXI", Spanish in Context 12 (2), 255-279.

Cestero Mancera, A. M. y Paredes García, F. (2018a). "Creencias y actitudes hacia las variedades cultas del español actual: el proyecto PRECAVES XXI”, Boletín de Filología, 53, 2, 11-43.

Cestero Mancera, A. M. y Paredes García, F. (2018b). "Creencias y actitudes de los jóvenes universitarios del centro-norte de España hacia las variedades cultas del español", Boletín de Filología, 53, 2, 45-86.

Crespo Cano, V. y Manjón-Cabeza Cruz, A. (1996). "Aproximación a las actitudes y creencias lingüísticas de futuros maestros", Docencia e Investigación, 21, 41-76.

García Marcos, F. (2008). Bases de planificación lingüistica de Andalucía, Almería: Univ. de Almería. García Marcos, F. (2015). Sociolingüistica. Madrid, Síntesis.

García Marcos, F. y Fuentes González, A. D. (1996). Mecanismos de prestigio y repercusión sociolingüistica, Almería: Univ. de Almería.

García Marcos, F. y Manjón-Cabeza Cruz, A. (1989). "Creencias lingüísticas en la E.G.B. Contribución a una política lingüística en Andalucía". En Actas del V Congreso Nacional de AESLA. Pamplona: Univ. de Navarra, 243-249.

De Molina Redondo, J. A. (1985). "Las lenguas de España después de la Constitución de 1978: el andaluz (sintaxis)". Revista de filología románica, 3, 69-76.

Díaz Salgado, L. C. (2002). "Creencias y actitudes sobre usos fónicos 'innovadores' del andaluz en los periodistas sevillanos de Canal Sur Televisión”, Tonos Digital 3.

Fontanella de Weinberg, M. B. (1979). "Dinámica social de un cambio lingüístico. La reestructuración de las palatales en el español bonaerense", México, CLH-IIF de la UNAM.

Fuentes González, A. D. (1996). Actitudes sociolingüisticas de la ciudad de Almería. Almería: GRUSTA.

Hernández Cabrera, C. y Samper Hernández, M. (2018). “Creencias y actitudes de los jóvenes universitarios canarios hacia las variedades cultas del español", Boletín de Filología, 53, 2, 179208.

Garvin P y Mathiot, M. (1974). "La urbanización del idioma guaraní. Problema de lengua y cultura". En P. Garvin y Lastra, Y. (eds.), Antología de estudios de etnolingüistica y sociolingüistica, México: UNAM, 303-313.

González- Rátiva, M. C., Muñoz-Builes, D., Guzmán García, M. F. y Correa Lopera, L. M. (2018). "Creencias y actitudes lingüísticas de los jóvenes universitarios antioqueños hacia las variedades normativas del español”, Boletín de Filología, 53, 2, 209-235.

Guerrero, S. y San Martín, A. (2018). "Creencias y actitudes de los jóvenes universitarios chilenos hacia las variedades cultas del español”, Boletín de Filología, 53, 2, 237-262.

Gutiérrez Böhmer, S. y Borzi, C. (2018). "Creencias y actitudes de los jóvenes universitarios 
rioplatenses hacia las variedades cultas del español”, Boletín de Filología, 53, 2, 263-292.

López Morales, H. (1989). Sociolingüistica. Madrid: Gredos.

Manjón-Cabeza Cruz, A. (2000). "Actitudes de los transmisores del prestigio lingüístico en Toledo: primeros datos", Docencia e Investigación, 25, 89-123.

Manjón-Cabeza Cruz, A. (2018a). "Creencias y actitudes de los jóvenes universitarios granadinos hacia las variedades cultas del español”, Boletín de Filología, 53, 2, 145-177.

Manjón-Cabeza Cruz, A. (2018b). "Una acomodación lingüística problemática: los diminutivos en los emigrantes ecuatorianos en Granada (España)", Itinerarios, 28, 67-80.

Martínez, M. y Moya Corral, J. A. (2000). "Reacciones actitudinales hacia la variación dialectal en hablantes granadinos”. Lingüística Española Actual, XXII/2, 137-156.

Méndez Guerrero, B. (2018). "Creencias y actitudes de los jóvenes universitarios mallorquines hacia las variedades cultas del español”, Boletín de Filología, 53, 2, 87-114.

Mondéjar Cumpián, J. (2006). Bibliografía sistemática y cronológica de las hablas andaluzas. Universidad de Málaga / Universidad de Granada. $2^{\mathrm{a}}$ ed.

Moreno Cabrera, J. C. (2006). La dignidad e igualdad de las lenguas. Crítica de la discriminación lingüística. Madrid: Alianza.

Moreno Fernández, F. (1998). Principios de sociolingüistica y sociología del lenguaje, Barcelona: Ariel.

Moreno Fernández, F. (2009). La lengua española en su geografía. Madrid: Arco/Libros.

Santana Marrero, J. (2018a). "Creencias y actitudes de jóvenes universitarios sevillanos hacia las variedades normativas del español de España: andaluza, canaria y castellana", Pragmática Sociocultural / Sociocultural Pragmatics, 6, 1, 71-97.

Santana Marrero, J. (2018b). "Creencias y actitudes de los jóvenes universitarios sevillanos hacia las variedades cultas del español”, Boletín de Filología, 53, 2, 115-144.

Silva-Corvalán, C. (2001). Sociolingüistica y pragmática del español, Washington, D.C.: Georgetown University Press.

Vaz de Soto, J. M. (1981). Defensa del habla andaluza. Sevilla: Edisur.

Villena Ponsoda, J. A. (2006). "Andaluz oriental y andaluz occidental: estandarización y planificación en ¿una o dos comunidades de habla?". En Cestero, A., Molina, I. y Paredes, F. (eds.). Estudios sociolingüisticos del español de España y América, Madrid: Arco/Libros, 233-254.

Yraola, A. (2014). "Actitudes lingüísticas en España”. En Chiquito, A. B. y Quesada Pacheco, M. A. (eds.). Actitudes lingüísticas de los hispanohablantes hacia el idioma español y sus variantes, Bergen Language and Linguistic Studies, 5, 551-636. 